\title{
Progress and Problems in Nutraceuticals Delivery
}

\section{Noha M. Zaki*}

Department of Pharmaceutics and Pharmaceutical Technology, College of Pharmacy, Taif University, Taif, KSA

\begin{abstract}
Nutraceuticals have always been considered as natural and safe supplements that may prevent disease, may substitute prescription drugs, may compensate for a poor diet or promote health. Several nutraceuticals products are in the market at present most of which are antioxidants and probiotics. The majority of nutraceutical products are intended for oral administration however; concerns related to their formulation, bioavailability and/or their site specific delivery arise. The labile nature, oral absorption and target-ability are the major underlying causes for poor nutraceuticals oral delivery. Investigators attempted to circumvent these obstacles by wide spectrum approaches tailored for each specific nutraceutical with solubility-enhancement techniques being the most commonly tackled. Recently, nanosizing techniques are utilized to answer the problem of nutraceuticals delivery with promising results in terms of nutraceutical-protection and stability on shelf and in vivo, solubility and dissolution rate enhancement, intestinal permeability improvement, intracellular and subcellular organelle targeting as well as extended circulation half-life all of which result in boosted bioavailability. The present review elucidates the progress and problems in the oral delivery of nutraceuticals.
\end{abstract}

Keywords: Nutraceuticals; Delivery oral; Targeting; Solubility enhancement; Probiotics; Antioxidants; Nanotechnology; Bioavailability

The International Food Information Council (IFIC) has defined foods that afford health benefits beyond basic nutrition, as "functional foods." These can range from broccoli to fortified foods such as calcium-fortified orange juice, to soy-based products to dietary supplements [1]. The formulation of the dietary supplements, functional foods or herbal products into marketed medicinal products is known as "nutraceuticals"; a term which combines "nutrition" and "pharmaceuticals". The nutraceuticals have many therapeutic benefits, and are especially relevant as antifatigue or for preventing or delaying a number of age-related diseases, i.e. arthritis, cancer, metabolic and cardiovascular diseases, Alzheimer's, Huntington disease, osteoporosis, cataracts, brain disorders, etc. [2]. Examples of nutraceuticals widely used nowadays are the polyvitamins, omega-3 fatty acids, carotenoids, polyphenols (anthocyanins, proanthocyanidins, flavanones, isoflavones, and ellagic acid) [3]. The best characterized polyphenol member is Curcumin \& its derivatives which are extracted from plant turmeric (Curcuma longa, family Zingiberaceae) which exhibits antioxidant, anti-inflammatory, and anti-cancer properties. The latter effect was attributed to activation of apoptosis signaling (curcumin inhibits $\mathrm{Bcl} 2$ and activates Caspase 9 to induce apoptosis) and blockade of many cell proliferation signaling pathways, (such as MAP kinase pathway, AKT pathway and mTOR pathways) [4-7]. Another wonder nutrient is coenzyme Q10, an antioxidant a benzoquinone derivative endogenously produced by the human body [8]. It is contained in many foods such as liver, heart and muscles of beef, nuts, vegetables and fish [9]. Other beneficial phytochemical compound is thymoquinone, a potent therapeutic agent of Nigella Sativa, has proven antioxidant, antiinflammatory, bronchodilator and anticancer effect [10-12]. The latter is mediated through multiple mechanisms: anti-proliferation, apoptosis induction, cell cycle arrest, ROS generation and anti-metastasis/antiangiogenesis [10]. While it might be easy for a formulator to develop a pharmaceutical forms (tablets, powders, capsules, suppositories, etc.) containing food bioactives, it is quiet challenging to obtain a satisfactory bioavailability for such nutraceuticals. The bioavailability is often jeopardized by the low solubility, stability and/or permeability of the bioactive in the GIT. In case of curcumin, extremely low serum levels, limited tissue distribution, apparent rapid metabolism and short circulation half-life are the underlying causes of its low oral bioavailability [13]. On the other hand, the bioavailability of the lipophilic antioxidant coenzyme Q10 was challenged by its low aqueous solubility and slow dissolution rate in GI fluids furnished by its highly lipophilic character $(\log \mathrm{P}=21)$ [14]. Moreover, permeability is limited by its large molecular weight (863), P-glycoprotein efflux and active transport by a number of transporters (including peptide transporters (PEPT1), cation/camitine transporters (OCT1, OCTN1, OCTN2 and OCT3) and organic anion transporters (AE2 and MCTl) [15].

Another category of nutraceuticals that are quickly growing in the past two decades are the "Probiotics" (i.e. health-promoting bacteria); with global market value $\$ 32.6$ billion in $2014[16,17]$. The oral delivery of probiotics is hampered by the low instability of the bacteria in the GIT and consequent loss of viability under the effect of high acidity and bile salt concentrations. The problem of oral delivery of the nutraceuticals at acceptable bioavailability has been tackled by formulators with various degrees of success. For Probiotics delivery, the bacteria could be immobilized into a polymer matrix, which is a kind of enteric-system that remains intact in the stomach but degrades and dissolves in the intestine. The Probiotics Encapsulation Technology or "PET" has emerged recently aiming for the protection and safe formulation and delivery of the living probiotic cell. Conditions that maintain cell viability like biomaterial selection, solvent type and toxicity and choice of proper technology are of paramount concern. Pertaining to the biomaterial, natural and synthetic polymers are used; factors to be addressed are: (i) physicochemical properties (chemical composition, morphology, mechanical strength, stability in GI fluids; (ii) toxicity assay; (iii) manufacturing and sterilization processes [18]. The most common biomaterials used for probiotics encapsulation are alginate, chitosan, carrageenan, gelatin, whey proteins, cellulose acetate phthalate, locust bean gum and starches [19]. The techniques

*Corresponding author: Noha M. Zaki, Department of Pharmaceutics and Pharmaceutical Technology, College of Pharmacy, Taif University, Taif, KSA, Tel: +966 595230971; E-mail: noha@tu.edu.sa; anm1998@lycos.com

Received March 25, 2014; Accepted May 03, 2014; Published May 13, 2014

Citation: Zaki NM (2014) Progress and Problems in Nutraceuticals Delivery. J Bioequiv Availab 6: 075-077. doi:10.4172/jbb.1000183

Copyright: () $2014 \mathrm{Lu} \mathrm{Y,} \mathrm{et} \mathrm{al.} \mathrm{This} \mathrm{is} \mathrm{an} \mathrm{open-access} \mathrm{article} \mathrm{distributed} \mathrm{under}$ the terms of the Creative Commons Attribution License, which permits unrestricted use, distribution, and reproduction in any medium, provided the original author and source are credited. 
attempted are: spray-drying, freeze-drying, fluidized bed drying, extrusion and emulsification techniques. A subsequent challenge is to ascertain that the gastro-protected encapsulated probiotics are released in simulated intestinal fluid (SIF) in vitro, a step that must be preceded by development of a standard protocol that simulates conditions prevailing in the GIT [20].

The nutraceuticals of non-bacterial origin mentioned above Require different yet broad spectrum endeavors including: use of adjuvants/ absorption enhancers like piperine [5], bile salts and surfactants. As a continuation, lipid-based systems like liposomes and emulsifyable/ self-emulsified systems (SEDDS) were used. SEDDs are particularly interesting because of the larger surface area afforded by the minute emulsion droplets, improved diffusion of the microscopic emulsion droplets, increased mucosal permeability due to surfactants and cosurfactants and improved lymphatic absorption (due to long-chain oils). The chain length dictates the route of transport. The molecules of long-chain fatty glycerides access the intestinal lymph in preference to the portal blood. The digestion products of long-chain triglycerides were preferentially resynthesized in the enterocyte, assembled into lipoproteins, and secreted into the mesenteric lymph, whereas medium chain triglycerides were primarily absorbed directly into the portal blood. The digested lipids of medium chain triglycerides with bile salts formlipophilic particles, and overcome the barrier of aqueous diffusion layer in GI tract [21]. In a different approach, solid dispersions and cyclodextrin complexation are promising in maximizing the effective surface area available for GI fluids and enhancing dissolution rate and oral absorption [22-24]. Figure 1 illustrates a decision tree on the formulation strategies to be tackled for poorly soluble nutraceuticals.

The advent of nanotechnology for pharmaceutical applications has opened a new avenue for stability, solubility and/or permeabilityenhancement of problematic nutraceuticals [25-27]. In this context, nanometric systems in absence or presence of carriers have been attempted. In absence of a carrier, nanonization of the bioactive, according to Noyes-Whitney equation, overcomes its "grease ball" nature and improves its wettability and dissolution rate. In presence of a carrier, an armory of nanocarriers is at hand for example: nanocapsules, micelles and nanoparticles, however, biocompatibility

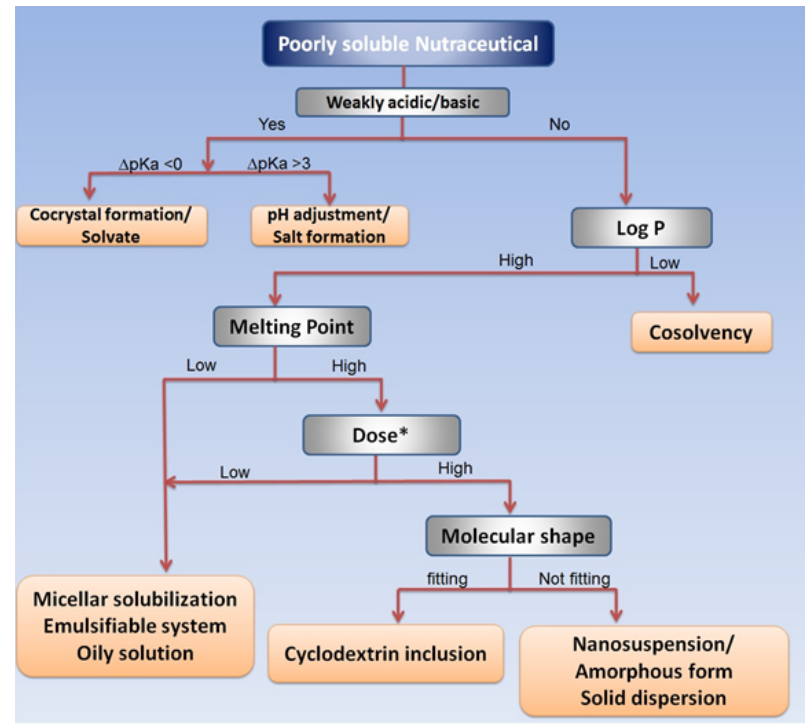

Figure 1: A decision tree for formulation of poorly-water soluble nutraceutical. and biodegradability-related issues are crucial. Biodegradable FDA-approved polymers e.g. polyesters are favored by formulators to develop nutraceutical-loaded nanoparticles. Polymer-based nanoparticles modulate the release of encapsulated bioactives, protect them from degradation, alter their biodistribution and shift their transport across biological membranes from a passive diffusion process to endocytosis one [28-31]. Additionally, targeting moiety can be fixed to nanoparticles surface [32-35]. The targeted and/or endocyticuptake of nanoparticles maximizes their intracellular delivery which is strictly needed to exert for example an anticancer effect [36-39]. Sub-cellular organelle targeting like mitochondrial-targeting (in case of coenzyme Q10) have been achieved by especial targeting moiety namely lipophilic triphenylphosphonium cation either chemically conjugated to the nanocarrier or the coenzyme Q10 [40], or resveratrol molecule [41]. Conjugating resveratrol to the membrane-permeable lipophilic triphenylphosphonium cation provided transient protection against metabolic conjugation, accumulated into mitochondria and was cytotoxic for fast-growing but not for slower-growing cells [41]. Such mitochondrial targeting of antioxidant nutraceuticals furnished a powerful tool to mediate mitochondrial and cellular redox processes of pathophysiological consequences [42]. The approach in which a bioactive is chemically linked to a polymer is well known as "Polymer Conjugates". The conjugates modulate the physicochemical, pharmacokinetic and therapeutic properties of the therapeutic agent. The water-soluble anticancer curcumin polyconjugates showed altered biodistribution and improved anticancer efficacy as it combines the dual advantage of enhanced aqueous solubility and polymer-mediated drug internalization [43].

For recapitulation, the consumer preferences and the advance in the field of functional foods, dietary supplements and phytochemical compounds triggered the search and implementation of novel formulation strategies to develop nutraceutical products to overcome low bioavailability and improve therapeutic efficacy.

\section{References}

1. http://wwwfoodinsightorg/Resources/Detailaspx?topic=2005

2. Gonzalez-Sarrias A, Larrosa M, Garcia-Conesa MT, Tomas-Barberan FA, Espin JC (2013) Nutraceuticals for older people: facts, fictions and gaps in knowledge. Maturitas 75: 313-334.

3. Espin JC, Garcia-Conesa MT, Tomas-Barberan FA (2007) Nutraceuticals: facts and fiction. Phytochemistry 68: 2986-3008.

4. Tang H, Murphy CJ, Zhang B, Shen Y, Van Kirk EA, et al. (2010) Curcumin polymers as anticancer conjugates. Biomaterials 31: 7139-7149.

5. Shaikh J, Ankola DD, Beniwal V, Singh D, Kumar MN (2009) Nanoparticle encapsulation improves oral bioavailability of curcumin by at least 9-fold when compared to curcumin administered with piperine as absorption enhancer. Eur J Pharm Sci 37: 223-230.

6. Prajakta D, Ratnesh J, Chandan K, Suresh S, Grace S, et al. (2009) Curcumin loaded $\mathrm{pH}$-sensitive nanoparticles for the treatment of colon cancer. J Biomed Nanotechnol 5: 445-455.

7. Narayanan NK, Nargi D, Randolph C, Narayanan BA (2009) Liposome encapsulation of curcumin and resveratrol in combination reduces prostate cancer incidence in PTEN knockout mice. Int J Cancer 125: 1-8.

8. Kendler BS (1999) Nutritional strategies in cardiovascular disease control: an update on vitamins and conditionally essential nutrients. Prog Cardiovasc Nurs 14: $124-129$.

9. Borek C (2006) Aging and antioxidants. Fruits and vegetables are powerful armor. Adv Nurse Pract 14: 35-38.

10. Woo CC, Kumar AP, Sethi G, Tan KH (2012) Thymoquinone: potential cure for inflammatory disorders and cancer. Biochem Pharmacol 83: 443-451. 
11. Singh A, Ahmad I, Akhter S, Jain GK, Iqbal Z, et al. (2013) Nanocarrier based formulation of Thymoquinone improves oral delivery: stability assessment, in vitro and in vivo studies. Colloids Surf B Biointerfaces 102: 822-832.

12. Shah M, Naseer MI, Choi MH, Kim MO, Yoon SC (2010)Amphiphilic PHA-mPEG copolymeric nanocontainers for drug delivery: preparation, characterization and in vitro evaluation. Int J Pharm 400: 165-175.

13. Anand P, Kunnumakkara AB, Newman RA, Aggarwal BB (2007) Bioavailability of curcumin: problems and promises. Mol Pharm 4: 807-818.

14. Balakrishnan P, Lee BJ, Oh DH, Kim JO, Lee YI, et al. (2009) Enhanced oral bioavailability of Coenzyme Q10 by self-emulsifying drug delivery systems. Int J Pharm 374: 66-72.

15. Palamakula A (2004) Biopharmaceutical classification and development of limonene-based self-nanoemulsified capsule dosage form of coenzyme q10. Texas Tech University Sciences Center 5: 55-79.

16. Cook MT, Tzortzis G, Charalampopoulos D, Khutoryanskiy VV (2012) Microencapsulation of probiotics for gastrointestinal delivery. J Control Release 162: 56-67.

17. Hafez MM, Maghrabi IA, Zaki NM (2013) Toward an alternative therapeutic approach for skin infections: Antagonistic activity of lactobacilli against antibiotic-resistant staphylococcus aureus and pseudomonas aeruginosa. Probiotics and Antimicrobial Proteins.

18. Gbassi GK, Vandamme T (2012) Probiotic encapsulation technology: from microencapsulation to release into the gut. Pharmaceutics 4: 149-163.

19. Chen S, Zhao Q, Ferguson LR, Shu Q, Weir I, et al. (2011) Development of a novel probiotic delivery system based on microencapsulation with protectants. Appl Microbiol Biotechnol .

20. Champagne CP (2012) Microencapsulation of probiotics in food: challenges and future prospects. Ther Deliv 3: 1249-1251.

21. New RRC, Kirby CJ (1999) Solubilisation aids. US Patent 5968549.

22. Yallapu MM, Jaggi M, Chauhan SC (2010) beta-Cyclodextrin-curcumin selfassembly enhances curcumin delivery in prostate cancer cells. Colloids Surf B Biointerfaces 79: 113-125.

23. Yallapu MM, Jaggi M, Chauhan SC (2010) Poly( $\left(^{2}\right.$-cyclodextrin)/curcumin selfassembly: a novel approach to improve curcumin delivery and its therapeutic efficacy in prostate cancer cells. Macromol Biosci 10: 1141-1151.

24. Abu-Dahab R, Odeh F2, Ismail SI3, Azzam H4, Al Bawab A2 (2013) Preparation, characterization and antiproliferative activity of thymoquinone-beta-cyclodextrin self assembling nanoparticles. Pharmazie 68: 939-944.

25. Yallapu MM, Jaggi M, Chauhan SC (2013) Curcumin nanomedicine: a road to cancer therapeutics. Curr Pharm Des 19: 1994-2010.

26. Yallapu MM, Jaggi M, Chauhan SC (2012) Curcumin nanoformulations: a future nanomedicine for cancer. Drug Discov Today 17: 71-80.

27. Yallapu MM, Jaggi M, Chauhan SC (2010) Scope of nanotechnology in ovarian cancer therapeutics. J Ovarian Res 3: 19.

28. Song XR, Cai Z, Zheng Y, He G, Cui FY, et al. (2009) Reversion of multidrug resistance by co-encapsulation of vincristine and verapamil in PLGA nanoparticles. Eur J Pharm Sci 37: 300-305.

29. Ravindran J, Nair HB, Sung B, Prasad S, Tekmal RR, et al. (2010) Thymoquinone poly (lactide-co-glycolide) nanoparticles exhibit enhanced antiproliferative, anti-inflammatory, and chemosensitization potential. Biochem Pharmacol 79: 1640-1647.

30. Patil Y, Panyam J (2009) Polymeric nanoparticles for siRNA delivery and gene silencing. Int J Pharm 367: 195-203.

31. Mundargi RC, Babu VR, Rangaswamy V, Patel P, Aminabhavi TM (2008) Nano/ micro technologies for delivering macromolecular therapeutics using poly(D,Llactide-co-glycolide) and its derivatives. J Control Release 125: 193-209.

32. Chavanpatil MD, Patil Y, Panyam J (2006) Susceptibility of nanoparticleencapsulated paclitaxel to P-glycoprotein-mediated drug efflux. Int J Pharm 320: 150-156.
33. Chan JM, Zhang L, Yuet KP, Liao G, Rhee JW, et al. (2009) PLGA-lecithin-PEG core-shell nanoparticles for controlled drug delivery. Biomaterials 30: 16271634.

34. Cegnar M, Kos J, Kristl J (2006) Intracellular delivery of cysteine protease inhibitor cystatin by polymeric nanoparticles. J Nanosci Nanotechnol 6: 30873094.

35. Acharya S, Dilnawaz F, Sahoo SK (2009) Targeted epidermal growth factor receptor nanoparticle bioconjugates for breast cancer therapy. Biomaterials 30 : $5737-5750$.

36. Zaki NM, Tirelli N (2011) Assessment of nanomaterials cytotoxicity and internalization. Methods Mol Biol 695: 243-259.

37. Zaki NM, Tirelli N (2010) Gateways for the intracellular access of nanocarriers: a review of receptor-mediated endocytosis mechanisms and of strategies in receptor targeting. Expert Opin Drug Deliv 7: 895-913.

38. Zaki NM, Nasti A, Tirelli N (2011) Nanocarriers for cytoplasmic delivery: cellular uptake and intracellular fate of chitosan and hyaluronic acid-coated chitosan nanoparticles in a phagocytic cell model. Macromol Biosci 11: 1747-1760.

39. Zaki NM (2013) Augmented cytotoxicity of hydroxycamptothecin-loaded nanoparticles in lung and colon cancer cells by chemosensitizing pharmaceutical excipients. Drug Deliv

40. CochemÃ@ HM1, Kelso GF, James AM, Ross MF, Trnka J, et al. (2007) Mitochondrial targeting of quinones: therapeutic implications. Mitochondrion 7 Suppl: S94-102.

41. Biasutto L, Mattarei A, Marotta E, Bradaschia A, Sassi N, et al. (2008) Development of mitochondria-targeted derivatives of resveratrol. Bioorg Med Chem Lett 18: 5594-5597.

42. Porteous CM, Logan A, Evans C, Ledgerwood EC, Menon DK, et al. (2010) Rapid uptake of lipophilic triphenylphosphonium cations by mitochondria in vivo following intravenous injection: implications for mitochondria-specific therapies and probes. Biochim Biophys Acta 1800: 1009-1017.

43. Safavy A, Raisch KP, Mantena S, Sanford LL, Sham SW, et al. (2007) Design and development of water-soluble curcumin conjugates as potential anticancer agents. J Med Chem 50: 6284-6288. 